\title{
Existence Criteria for Singular Initial Value Problems with Sign Changing Nonlinearities
}

\author{
RAVI P. AGARWAL ${ }^{a, *}$, DONAL O'REGAN ${ }^{b}$ \\ and $V$. LAKSHMIKANTHAM ${ }^{a}$ \\ ${ }^{a}$ Department of Mathematical Sciences, Florida Institute of Technology, Melbourne, \\ Florida 32901, USA; ${ }^{\mathrm{b}}$ Department of Mathematics, National University of Ireland, \\ Galway, Ireland;
}

(Received 16 April 2001)

A general existence theory is presented for initial value problems where our nonlinearity may be singular in its dependent variable and may also change sign.

Keywords: Singular initial value problem; Sign changing nonlinearity; Upper and lower solutions; Existence criteria

\section{INTRODUCTION}

This paper discusses the singular initial value problem

$$
\left\{\begin{array}{l}
y^{\prime}=q(t) f(t, y), \quad 0<t<T(<\infty) \\
y(0)=0
\end{array}\right.
$$

where our nonlinearity $f$ is allowed to change sign. In addition $f$ may not be a Carathéodory function because of the singular behavior of the $y$ variable i.e. $f$ may be singular at $y=0$. Nonsingular problems have been discussed extensively in the literature [1-6]. However only a few papers $[2,3]$ have appeared when the nonlinearity $f$ is singular at $y=0$.

\footnotetext{
* Corresponding author.
} 
The results here are new and they complement and extend those in $[2,3]$. In this paper to establish existence for (1.1) we approximate (1.1) by a sequence of nonsingular problems, each of which has a lower solution $\alpha$ and a upper solution $\beta_{n}$. Using the Schauder fixed point theorem we establish the existence of a solution which lies between $\alpha$ and $\beta_{n}$ for each approximating problem. The Arzela-Ascoli theorem will then complete the proof. In addition we also present, in this paper, easily verifiable criteria which guarantee that (1.1) has a solution $y \in C[0, T]$ with $y>0$ on $(0, T]$.

\section{EXISTENCE THEORY}

In this section we discuss the initial value problem

$$
\left\{\begin{array}{l}
y^{\prime}=q(t) f(t, y), \quad 0<t<T(<\infty) \\
y(0)=0
\end{array}\right.
$$

where our nonlinearity $f$ may change sign. We first present an upper and lower solution result for the singular initial value problem. The idea involves approximating (1.1) by a sequence of nonsingular problems each of which has a lower solution $\alpha$ and a upper solution $\beta_{n}$. The Arzela Ascoli theorem will then complete the proof. After the proof we discuss how to construct the lower solution $\alpha$. In particular general criteria will be given which will enable us to verify immediately that a particular equation has a lower solution $\alpha$. This has the added advantage that we do not need to construct $\alpha$ explicitly for each example. Also in this section we replace the $\beta_{n}$ condition with another more easily verifiable one. Examples will then be given to illustrate our theory.

THEOREM 2.1 Let $n_{0} \in\{3,4, \ldots\}$ be fixed and suppose the following conditions are satisfied:

$$
\begin{gathered}
f:[0, T] \times(0, \infty) \rightarrow \mathbf{R} \text { is continuous, } \\
q \in C(0, T], q>0 \text { on }(0, T] \text { and } \int_{0}^{T} q(x) \mathrm{d} x<\infty,
\end{gathered}
$$




$$
\begin{aligned}
& \left\{\begin{array}{l}
\text { let } n \in N_{0}=\left\{n_{0}, n_{0}+1, \ldots\right\} \text { and associated with each } n \\
\text { we have a constant } \rho_{n} \text { such that }\left\{\rho_{n}\right\} \text { is a nonincreasing } \\
\text { sequence with } \lim _{n \rightarrow \infty} \rho_{n}=0 \text { and such that for } \\
\frac{T}{n} \leqslant t \leqslant T \text { we have } q(t) f\left(t, \rho_{n}\right) \geqslant 0,
\end{array}\right. \\
& \left\{\begin{array}{l}
\exists \alpha \in C[0, T] \cap C^{\mathrm{l}}(0, T], \alpha(0)=0, \alpha>0 \text { on }(0, T] \\
\text { such that for each } n \in N_{0}, q(t) f(t, \alpha(t)) \geqslant \alpha^{\prime}(t) \text { for } \\
t \in\left[\frac{T}{n}, T\right) \text { and } q(t) f\left(\frac{T}{n}, \alpha(t)\right) \geqslant \alpha^{\prime}(t) \text { for } t \in\left(0, \frac{T}{n}\right),
\end{array}\right.
\end{aligned}
$$

$$
\left\{\begin{array}{l}
\text { for each } n \in N_{0}, \exists \beta_{n} \in C[0, T] \cap C^{1}(0, T] \text { with } \\
\beta_{n}(t) \geqslant \alpha(t) \text { and } \beta_{n}(t) \geqslant \rho_{n} \text { for } t \in[0, T] \text { and } \\
q(t) f\left(t, \beta_{n}(t)\right) \leqslant \beta_{n}^{\prime}(t) \text { for } t \in\left[\frac{T}{n}, T\right) \text { with } \\
q(t) f\left(\frac{T}{n}, \beta_{n}(t)\right) \leqslant \beta_{n}^{\prime}(t) \text { for } t \in\left(0, \frac{T}{n}\right), \\
a_{0} \equiv \max \left\{\sup _{t \in[0, T]} \beta_{n}(t): n \in N_{0}\right\}<\infty
\end{array}\right.
$$

and

$$
\left\{\begin{array}{l}
|f(t, y)| \leqslant g(y) \text { on }[0, T] \times\left(0, a_{0}\right] \text { with } \\
g>0 \text { continuous and nonincreasing on }(0, \infty)
\end{array}\right.
$$

Then (2.1) has a solution $y \in C[0, T] \cap C^{1}(0, T]$ with $y(t) \geqslant \alpha(t)$ for $t \in[0, T]$.

Proof Without loss of generality assume $\rho_{n_{0}} \leqslant \min _{t \in[T / 3, T]} \alpha(t)$. Fix $n \in N_{0}$. Let $t_{n} \in[0, T / 3]$ be such that

$$
\alpha\left(t_{n}\right)=\rho_{n} \text { and } \alpha(t) \leqslant \rho_{n} \text { for } t \in\left[0, t_{n}\right] .
$$

Define

$$
\alpha_{n}(t)= \begin{cases}\rho_{n} & \text { if } t \in\left[0, t_{n}\right] \\ \alpha(t) & \text { if } t \in\left(t_{n}, T\right]\end{cases}
$$


Consider the initial value problem

$$
\left\{\begin{array}{l}
y^{\prime}=q(t) f_{n}^{*}(t, y), \quad 0<t<T \\
y(0)=\rho_{n}
\end{array}\right.
$$

here

$$
f_{n}^{*}(t, y)=\left\{\begin{array}{l}
f\left(\frac{T}{n}, \beta_{n}(t)\right), \quad y \geqslant \beta_{n}(t) \text { and } 0 \leqslant t \leqslant \frac{T}{n} \\
f\left(t, \beta_{n}(t)\right), \quad y \geqslant \beta_{n}(t) \text { and } \frac{T}{n} \leqslant t \leqslant T \\
f\left(\frac{T}{n}, y\right), \quad \alpha_{n}(t) \leqslant y \leqslant \beta_{n}(t) \text { and } 0 \leqslant t \leqslant \frac{T}{n} \\
f(t, y), \quad \alpha_{n}(t) \leqslant y \leqslant \beta_{n}(t) \text { and } \frac{T}{n} \leqslant t \leqslant T \\
f\left(t, \alpha_{n}(t)\right), \quad y \leqslant \alpha_{n}(t) \text { and } \frac{T}{n} \leqslant t \leqslant T \\
f\left(\frac{T}{n}, \alpha_{n}(t)\right), \quad y \leqslant \alpha_{n}(t) \text { and } 0 \leqslant t \leqslant \frac{T}{n} .
\end{array}\right.
$$

Schauder's fixed point theorem $[1,2]$ guarantees that $(2.9)^{n}$ has a solution $y_{n} \in C[0, T] \cap C^{\mathrm{l}}(0, T]$. We first show

$$
y_{n}(t) \geqslant \alpha_{n}(t) \text { for } t \in[0, T]
$$

Suppose (2.10) is not true. Then there exists $\tau_{1}<\tau_{2} \in[0, T]$ with

$$
y_{n}\left(\tau_{1}\right)=\alpha_{n}\left(\tau_{1}\right), \quad y_{n}\left(\tau_{2}\right)<\alpha_{n}\left(\tau_{2}\right)
$$

and

$$
y_{n}(t)<\alpha_{n}(t) \text { for } t \in\left(\tau_{1}, \tau_{2}\right)
$$

Of course

$$
y_{n}\left(\tau_{2}\right)-\alpha_{n}\left(\tau_{2}\right)=\int_{\tau_{1}}^{\tau_{2}}\left(y_{n}-\alpha_{n}\right)^{\prime}(t) \mathrm{d} t
$$


We now claim

$$
\left(y_{n}-\alpha_{n}\right)^{\prime}(t) \geqslant 0 \text { and a.e. } t \in\left(\tau_{1}, \tau_{2}\right) \text {. }
$$

If (2.12) is true then (2.11) implies

$$
y_{n}\left(\tau_{2}\right)-\alpha_{n}\left(\tau_{2}\right) \geqslant 0
$$

a contradiction. As a result if we show (2.12) is true then (2.10) will follow. To see (2.12) we will in fact prove more i.e. we will show

$$
\left(y_{n}-\alpha_{n}\right)^{\prime}(t) \geqslant 0 \text { for } t \in\left(\tau_{1}, \tau_{2}\right) \text { provided } t \neq t_{n} \text {. }
$$

Fix $t \in\left(\tau_{1}, \tau_{2}\right)$ and assume $t \neq t_{n}$. Then $y_{n}(t)-\alpha_{n}(t)<0$. Now either (i) $t<t_{n}$; or (ii) $t>t_{n}$.

Case (i) $t<t_{n}(\leqslant T / 3)$.

First suppose $t_{n} \geqslant T / n$. Then

$$
\begin{aligned}
\left(y_{n}-\alpha_{n}\right)^{\prime}(t) & =\left[q(t) f_{n}^{*}\left(t, y_{n}(t)\right)-\alpha_{n}^{\prime}(t)\right] \\
& =\left\{\begin{array}{l}
q(t) f\left(\frac{T}{n}, \alpha_{n}(t)\right)-\alpha_{n}^{\prime}(t), \quad 0<t \leqslant \frac{T}{n} \\
q(t) f\left(t, \alpha_{n}(t)\right)-\alpha_{n}^{\prime}(t), \quad \frac{T}{n} \leqslant t<t_{n}
\end{array}\right. \\
& =\left\{\begin{array}{l}
q(t) f\left(\frac{T}{n}, \rho_{n}\right), \quad 0<t \leqslant \frac{T}{n} \\
q(t) f\left(t, \rho_{n}\right), \quad \frac{T}{n} \leqslant t<t_{n}
\end{array}\right. \\
& \geqslant 0,
\end{aligned}
$$

from (2.4). Next suppose $t_{n} \leqslant T / n$. Then $t \leqslant T / n$ so we have

$$
\left(y_{n}-\alpha_{n}\right)^{\prime}(t)=q(t) f\left(\frac{T}{n}, \alpha_{n}(t)\right)-\alpha_{n}^{\prime}(t)=q(t) f\left(\frac{T}{n}, \rho_{n}\right) \geqslant 0
$$

from (2.4). 
Case (ii) $t>t_{n}$.

First suppose $t_{n} \leqslant T / n$. Then

$$
\begin{aligned}
\left(y_{n}-\alpha_{n}\right)^{\prime}(t) & =\left[q(t) f_{n}^{*}\left(t, y_{n}(t)\right)-\alpha_{n}^{\prime}(t)\right] \\
& =\left\{\begin{array}{l}
q(t) f\left(\frac{T}{n}, \alpha_{n}(t)\right)-\alpha_{n}^{\prime}(t), \quad t_{n}<t \leqslant \frac{T}{n} \\
q(t) f\left(t, \alpha_{n}(t)\right)-\alpha_{n}^{\prime}(t), \quad \frac{T}{n} \leqslant t
\end{array}\right. \\
& =\left\{\begin{array}{l}
q(t) f\left(\frac{T}{n}, \alpha(t)\right)-\alpha^{\prime}(t), \quad t_{n}<t \leqslant \frac{T}{n} \\
q(t) f(t, \alpha(t))-\alpha^{\prime}(t), \quad \frac{T}{n} \leqslant t
\end{array}\right. \\
& \geqslant 0,
\end{aligned}
$$

from (2.5). Next suppose $t_{n} \geqslant T / n$. Then

$$
\left(y_{n}-\alpha_{n}\right)^{\prime}(t)=q(t) f\left(t, \alpha_{n}(t)\right)-\alpha_{n}^{\prime}(t)=q(t) f(t, \alpha(t))-\alpha^{\prime}(t) \geqslant 0,
$$

from (2.5).

Consequently (2.12) (and so (2.10)) is true, and now since $\alpha(t) \leqslant \alpha_{n}(t)$ for $t \in[0, T]$ we have

$$
\alpha(t) \leqslant \alpha_{n}(t) \leqslant y_{n}(t) \quad \text { for } t \in[0, T] .
$$

Next we show

$$
y_{n}(t) \leqslant \beta_{n}(t) \text { for } t \in[0, T]
$$

If (2.14) is not true then there exists $\tau_{1}<\tau_{2} \in[0, T]$ with

$$
y_{n}\left(\tau_{1}\right)=\beta_{n}\left(\tau_{1}\right), y_{n}\left(\tau_{2}\right)>\beta_{n}\left(\tau_{2}\right)
$$

and

$$
y_{n}(t)>\beta_{n}(t) \text { for } t \in\left(\tau_{1}, \tau_{2}\right) .
$$


Notice also that

$$
y_{n}\left(\tau_{2}\right)-y_{n}\left(\tau_{1}\right)=\int_{\tau_{1}}^{\tau_{2}} q(s) f_{n}^{*}\left(s, y_{n}(s)\right) \mathrm{d} s
$$

There are three cases to consider, namely (i) $T / n \leqslant \tau_{1}$; $\tau_{1}<\tau_{2} \leqslant T / n$; and (iii) $\tau_{1}<T / n<\tau_{2}$.

Case (i) $T / n \leqslant \tau_{1}$.

Then (2.6) implies

$$
\begin{aligned}
y_{n}\left(\tau_{2}\right)-y_{n}\left(\tau_{1}\right) & =\int_{\tau_{1}}^{\tau_{2}} q(s) f\left(s, \beta_{n}(s)\right) \mathrm{d} s \leqslant \int_{\tau_{1}}^{\tau_{2}} \beta_{n}^{\prime}(s) \mathrm{d} s \\
& =\beta_{n}\left(\tau_{2}\right)-\beta_{n}\left(\tau_{1}\right),
\end{aligned}
$$

a contradiction.

Case (ii) $\tau_{1}<\tau_{2} \leqslant T / n$.

Then (2.6) implies

$$
\begin{aligned}
y_{n}\left(\tau_{2}\right)-y_{n}\left(\tau_{1}\right) & =\int_{\tau_{1}}^{\tau_{2}} q(s) f\left(\frac{T}{n}, \beta_{n}(s)\right) \mathrm{d} s \\
& \leqslant \int_{\tau_{1}}^{\tau_{2}} \beta_{n}^{\prime}(s) \mathrm{d} s=\beta_{n}\left(\tau_{2}\right)-\beta_{n}\left(\tau_{1}\right),
\end{aligned}
$$

a contradiction.

Case (iii) $\tau_{1}<T / n<\tau_{2}$. Now

$$
y_{n}\left(\frac{T}{n}\right)-y_{n}\left(\tau_{1}\right)=\int_{\tau_{1}}^{T / n} q(s) f\left(\frac{T}{n}, \beta_{n}(s)\right) \mathrm{d} s \leqslant \beta_{n}\left(\frac{T}{n}\right)-\beta_{n}\left(\tau_{1}\right)
$$

and

$$
y_{n}\left(\tau_{2}\right)-y_{n}\left(\frac{T}{n}\right)=\int_{T / n}^{\tau_{2}} q(s) f\left(s, \beta_{n}(s)\right) \mathrm{d} s \leqslant \beta_{n}\left(\tau_{2}\right)-\beta_{n}\left(\frac{T}{n}\right) .
$$


Combine to obtain

$$
y_{n}\left(\tau_{2}\right)-y_{n}\left(\tau_{1}\right) \leqslant \beta_{n}\left(\tau_{2}\right)-\beta_{n}\left(\tau_{1}\right)
$$

a contradiction.

Thus (2.14) holds. In particular for $t \in[0, T]$ we have

$$
\alpha(t) \leqslant \alpha_{n}(t) \leqslant y_{n}(t) \leqslant \beta_{n}(t) \leqslant a_{0}
$$

here $a_{0}$ is given in (2.7). We next show

$$
\left\{y_{n}\right\}_{n \in N_{0}} \text { is a bounded, equicontinuous family on }[0, T] \text {. }
$$

To see this notice (2.8) and (2.15) guarantee that we have

$$
\frac{\left|y_{n}^{\prime}(t)\right|}{g\left(y_{n}(t)\right)} \leqslant q(t) \quad \text { for } t \in(0, T)
$$

and so

$$
\pm v_{n}^{\prime}(t) \leqslant q(t) \quad \text { for } t \in(0, T)
$$

here

$$
v_{n}(t)=\int_{0}^{y_{n}(t)} \frac{\mathrm{d} u}{g(u)}=G\left(y_{n}(t)\right) .
$$

For $t, s \in[0, T]$ we have

$$
\left|v_{n}(t)-v_{n}(s)\right|=\left|\int_{s}^{t} v_{n}^{\prime}(\tau) \mathrm{d} \tau\right| \leqslant\left|\int_{s}^{t} q(\tau) \mathrm{d} \tau\right| .
$$

This together with the uniform continuity of $G^{-1}$ on $\left[0, G\left(a_{0}\right)\right]$ and

$$
\begin{aligned}
\left|y_{n}(t)-y_{n}(s)\right| & =\left|G^{-1}\left(G\left(y_{n}(t)\right)\right)-G^{-1}\left(G\left(y_{n}(s)\right)\right)\right| \\
& =\left|G^{-1}\left(v_{n}(t)\right)-G^{-1}\left(v_{n}(s)\right)\right|
\end{aligned}
$$


immediately guarantees that $\left\{y_{n}\right\}_{n \in N_{0}}$ is equicontinuous on $[0, T]$. Thus (2.16) holds. The Arzela-Ascoli theorem guarantees the existence of a subsequence $N_{1}$ of $N_{0}$ and a function $y \in C[0, T]$ with $y_{n}$ converging uniformly on $[0, T]$ to $y$ as $n \rightarrow \infty$ through $N_{1}$. Also $y(0)=0$ and $\alpha(t) \leqslant y(t) \leqslant a_{0}$ for $t \in[0, T]$. Fix $t \in(0, T)$ and let $n_{1} \in N_{1}$ be such that $T / n_{1}<t<T$. Let $N_{1}^{*}=\left\{n \in N_{1}: n \geqslant n_{1}\right\}$. Now $y_{n}, n \in N_{1}^{*}$, satisfies

$$
\begin{aligned}
y_{n}(t) & =y_{n}(T)-\int_{t}^{T} q(s) f_{n}^{*}\left(s, y_{n}(s)\right) \mathrm{d} s \\
& =y_{n}(T)-\int_{t}^{T} q(s) f\left(s, y_{n}(s)\right) \mathrm{d} s .
\end{aligned}
$$

Let $n \rightarrow \infty$ through $N_{1}^{*}$ to obtain

$$
y(t)=y(T)-\int_{t}^{T} q(s) f(s, y(s)) \mathrm{d} s
$$

We can do this argument for each $t \in(0, T)$.

Remark 2.1 We could replace (2.7) and (2.8) in Theorem 2.1 with the following condition:

$$
\left\{\begin{array}{l}
\text { for each } t \in[0, T] \text {, we have that }\left\{\beta_{n}(t)\right\} \text { is a } \\
\text { nonincreasing sequence and } \lim _{n \rightarrow \infty} \beta_{n}(0)=0 .
\end{array}\right.
$$

To see this notice that we only needed (2.8) in the proof of Theorem 2.1 from (2.16) onwards. Here notice we have

$$
\alpha(t) \leqslant \alpha_{n}(t) \leqslant y_{n}(t) \leqslant \beta_{n}(t) \leqslant \beta_{n_{0}}(t) \text { for } t \in[0, T] .
$$

Now lets look at the interval $\left[T / n_{0}, T\right]$. Let

$$
R_{n_{0}}=\sup \left\{|q(x) f(x, y)|: x \in\left[\frac{T}{n_{0}}, T\right] \text { and } \alpha(x) \leqslant y \leqslant \beta_{n_{0}}(x)\right\} .
$$


We have immediately that

$$
\left\{y_{n}\right\}_{n=n_{0}}^{\infty} \text { is a bounded, equicontinuous family on }\left[T / n_{0}, T\right] \text {. }
$$

The Arzela-Ascoli theorem guarantees the existence of a subsequence $N_{n_{0}}$ of integers and a function $z_{n_{0}} \in C\left[T / n_{0}, T\right]$ with $y_{n}$ converging uniformly to $z_{n_{0}}$ on $\left[T / n_{0}, T\right]$ as $n \rightarrow \infty$ through $N_{n_{0}}$. Proceed inductively to obtain subsequences of integers

$$
N_{n_{0}} \supseteq N_{n_{0}+1} \supseteq \cdots \supseteq N_{k} \supseteq \cdots
$$

and functions

$$
z_{k} \in C\left[\frac{T}{k}, T\right]
$$

with

$y_{n}$ converging uniformly to $z_{k}$ on $[T / k, T]$ as $n \rightarrow \infty$ through $N_{k}$

and

$$
z_{k+1}=z_{k} \text { on }\left[\frac{T}{k}, T\right]
$$

Define a function $y:[0, T] \rightarrow[0, \infty)$ by $y(x)=z_{k}(x)$ on $[T / k, T]$ and $y(0)=0$. Notice $y$ is well defined and $\alpha(t) \leqslant y(t) \leqslant \beta_{n_{0}}(t)$ for $t \in(0, T)$. Fix $t \in(0, T)$ and let $m \in\left\{n_{0}, n_{0}+1, \ldots\right\}$ be such that $T / m<t<T$. Let $N_{m}^{*}=\left\{n \in N_{m}: n \geqslant m\right\}$. Now $y_{n}, n \in N_{m}^{*}$, satisfies

$$
y_{n}(t)=y_{n}(T)-\int_{t}^{T} q(s) f\left(s, y_{n}(s)\right) \mathrm{d} s .
$$

Let $n \rightarrow \infty$ through $N_{m}^{*}$ to obtain

$$
y(t)=y(T)-\int_{t}^{T} q(s) f(s, y(s)) \mathrm{d} s
$$


We can do this argument for each $t \in(0, T)$. It remains to show $y$ is continuous at 0 . Let $\epsilon>0$ be given. Now since $\lim _{n \rightarrow \infty} \beta_{n}(0)=0$ there exists $n_{1} \in\left\{n_{0}, n_{0}+1, \ldots\right\}$ with $\beta_{n_{1}}(0)<\epsilon / 2$. Since $\beta_{n_{1}} \in C[0, T]$ there exists $\delta_{n_{1}}>0$ with

$$
\beta_{n_{1}}(t)<\frac{c}{2} \quad \text { for } t \in\left[0, \delta_{n_{1}}\right]
$$

Now for $n \geqslant n_{1}$ we have, since $\left\{\beta_{n}(t)\right\}$ is nonincreasing for each $t \in[0, T]$,

$$
\beta_{n}(t) \leqslant \beta_{n_{1}}(t)<\frac{\epsilon}{2} \quad \text { for } t \in\left[0, \delta_{n_{1}}\right]
$$

This together with the fact that $\alpha(t) \leqslant y_{n}(t) \leqslant \beta_{n}(t)$ for $t \in[0, T]$, implies for $n \geqslant n_{1}$ that we have

$$
\alpha(t) \leqslant y_{n}(t)<\frac{\epsilon}{2} \quad \text { for } t \in\left[0, \delta_{n_{1}}\right]
$$

Consequently

$$
0 \leqslant \alpha(t) \leqslant y(t) \leqslant \frac{\epsilon}{2}<\epsilon \text { for } t \in\left(0, \delta_{n_{1}}\right]
$$

and so $y$ is continuous at 0 . Thus $y \in C[0, T]$.

Remark 2.2 Suppose (2.2)-(2.5), (2.7) and (2.8) hold, and in addition assume the following conditions are satisfied:

$$
\left\{\begin{array}{l}
\text { for each } n \in N_{0} \text { we have } q(t) f(t, y) \geqslant \alpha^{\prime}(t) \text { for } \\
(t, y) \in\left[\frac{T}{n}, T\right) \times\{y \in(0, \infty): y<\alpha(t)\} \text { and } \\
q(t) f\left(\frac{T}{n}, y\right) \geqslant \alpha^{\prime}(t) \text { for } \\
(t, y) \in\left(0, \frac{T}{n}\right) \times\{y \in(0, \infty): y<\alpha(t)\}
\end{array}\right.
$$


and

$$
\left\{\begin{array}{l}
\text { for each } n \in N_{0}, \exists \beta_{n} \in C[0, T] \cap C^{1}(0, T] \text { with } \\
\beta_{n}(t) \geqslant \rho_{n} \text { for } t \in[0, T] \text { and } q(t) f\left(t, \beta_{n}(t)\right) \leqslant \beta_{n}^{\prime}(t) \\
\text { for } t \in\left[\frac{T}{n}, T\right) \text { with } q(t) f\left(\frac{T}{n}, \beta_{n}(t)\right) \leqslant \beta_{n}^{\prime}(t) \\
\text { for } t \in\left(0, \frac{T}{n}\right) .
\end{array}\right.
$$

Then the result in Theorem 2.1 is again true. This follows immediately from Theorem 2.1 once we show (2.6) holds i.e. once we show $\beta_{n}(t) \geqslant \alpha(t)$ for $t \in[0, T]$ for each $n \in\left\{n_{0}, n_{0}+1, \ldots\right\}$. To see this suppose it is false for some $n \in\left\{n_{0}, n_{0}+1, \ldots\right\}$. Then there exists $\tau_{1}<\tau_{2} \in[0, T]$ with

$$
\beta_{n}\left(\tau_{1}\right)=\alpha\left(\tau_{1}\right), \beta_{n}\left(\tau_{2}\right)<\alpha\left(\tau_{2}\right) \text { and } \beta_{n}(t)<\alpha(t) \text { for } t \in\left(\tau_{1}, \tau_{2}\right) \text {. }
$$

There are three cases to consider, namely (i) $T / n \leqslant \tau_{1}$; (ii) $\tau_{1}<\tau_{2} \leqslant T / n$ and (iii) $\tau_{1}<T / n<\tau_{2}$.

Case (i) $T / n \leqslant \tau_{1}$.

Then (2.19) and $\beta_{n}(t)<\alpha(t)$ for $t \in\left(\tau_{1}, \tau_{2}\right)$ yields

$$
\begin{aligned}
\beta_{n}\left(\tau_{2}\right)-\beta_{n}\left(\tau_{1}\right) & =\int_{\tau_{1}}^{\tau_{2}} \beta_{n}^{\prime}(s) \mathrm{d} s \geqslant \int_{\tau_{1}}^{\tau_{2}} q(s) f\left(s, \beta_{n}(s)\right) \mathrm{d} s \\
& \geqslant \int_{\tau_{1}}^{\tau_{2}} \alpha^{\prime}(s) \mathrm{d} s=\alpha\left(\tau_{2}\right)-\alpha\left(\tau_{1}\right),
\end{aligned}
$$

a contradiction.

Case (ii) $\tau_{1}<\tau_{2} \leqslant T / n$. Then

$$
\begin{aligned}
\beta_{n}\left(\tau_{2}\right)-\beta_{n}\left(\tau_{1}\right) & =\int_{\tau_{1}}^{\tau_{2}} \beta_{n}^{\prime}(s) \mathrm{d} s \geqslant \int_{\tau_{1}}^{\tau_{2}} q(s) f\left(\frac{T}{n}, \beta_{n}(s)\right) \mathrm{d} s \\
& \geqslant \int_{\tau_{1}}^{\tau_{2}} \alpha^{\prime}(s) \mathrm{d} s=\alpha\left(\tau_{2}\right)-\alpha\left(\tau_{1}\right),
\end{aligned}
$$

a contradiction. 
Case (iii) $\tau_{1}<T / n<\tau_{2}$.

Then

$$
\beta_{n}\left(\frac{T}{n}\right)-\beta_{n}\left(\tau_{1}\right) \geqslant \int_{\tau_{1}}^{T / n} q(s) f\left(\frac{T}{n}, \beta_{n}(s)\right) \mathrm{d} s \geqslant \alpha\left(\frac{T}{n}\right)-\alpha\left(\tau_{1}\right),
$$

and

$$
\beta_{n}\left(\tau_{2}\right)-\beta_{n}\left(\frac{T}{n}\right) \geqslant \int_{T / n}^{\tau_{2}} q(s) f\left(s, \beta_{n}(s)\right) \mathrm{d} s \geqslant \alpha\left(\tau_{2}\right)-\alpha\left(\frac{T}{n}\right) .
$$

Combine to get

$$
\beta_{n}\left(\tau_{2}\right)-\beta_{n}\left(\tau_{1}\right) \geqslant \alpha\left(\tau_{2}\right)-\alpha\left(\tau_{1}\right)
$$

a contradiction.

If in (2.4) we replace $T / n \leqslant t \leqslant T$ by $0 \leqslant t \leqslant T$ then in this case we define $f_{n}^{*}$ as follows:

$$
f_{n}^{*}(t, y)= \begin{cases}f\left(t, \beta_{n}(t)\right), & y \geqslant \beta_{n}(t) \\ f(t, y), & \alpha_{n}(t) \leqslant y \leqslant \beta_{n}(t) \\ f\left(t, \alpha_{n}(t)\right), & y \leqslant \alpha_{n}(t) .\end{cases}
$$

For completeness we state the result.

THEOREM 2.2 Suppose (2.2) and (2.3) hold. In addition assume the following conditions hold

$$
\begin{gathered}
\left\{\begin{array}{l}
\text { let } n \in\{1,2, \ldots\}=N_{1} \text { and associated with each } \\
n \in N_{1} \text { we have a constant } \rho_{n} \text { such that }\left\{\rho_{n}\right\}, \\
\text { is a nonincreasing sequence with } \lim _{n \rightarrow \infty} \rho_{n}=0 \\
\text { and such that for } 0 \leqslant t \leqslant T \text { we have } q(t) f\left(t, \rho_{n}\right) \geqslant 0,
\end{array}\right. \\
\left\{\begin{array}{l}
\exists \alpha \in C[0, T] \cap C^{1}(0, T], \alpha(0)=0, \alpha>0 \text { on }(0, T] \\
\text { such that } q(t) f(t, \alpha(t)) \geqslant \alpha^{\prime}(t) \text { for } t \in(0, T)
\end{array}\right.
\end{gathered}
$$


and

$$
\left\{\begin{array}{l}
\text { for each } n \in N_{1}, \exists \beta_{n} \in C[0, T] \cap C^{1}(0, T] \\
\text { with } \beta_{n}(t) \geqslant \alpha(t) \text { and } \beta_{n}(t) \geqslant \rho_{n} \text { for } t \in[0, T] \\
\text { and } q(t) f\left(t, \beta_{n}(t)\right) \leqslant \beta_{n}^{\prime}(t) \text { for } t \in(0, T)
\end{array}\right.
$$

Finally assume either (2.17) or (2.7), (2.8) (with $N_{0}$ replaced by $N_{1}$ ) occur. Then (2.1) has a solution $y \in C[0, T] \cap C^{\prime}(0, T]$ with $y(t) \geqslant \alpha(t)$ for $t \in[0, T]$.

Next we discuss how to construct the lower solution $\alpha$ in (2.5) (and in (2.18)). Suppose the following condition is satisfied:

$$
\left\{\begin{array}{l}
\text { let } n \in N_{0} \text { and associated with each } n \text { we } \\
\text { have a constant } \rho_{n} \text { such that }\left\{\rho_{n}\right\} \text { is a decreasing } \\
\text { sequence with } \lim _{n \rightarrow \infty} \rho_{n}=0 \text { and there exists a } \\
\text { constant } k_{0}>0 \text { such that for } \frac{T}{n} \leqslant t \leqslant T \\
\text { and } 0<y \leqslant \rho_{n} \text { we have } q(t) f(t, y) \geqslant k_{0} .
\end{array}\right.
$$

The argument in [2, Chapter 1] guarantees that there exists a $\alpha \in C[0, T] \cap C^{\prime}(0, T], \alpha(0)=0, \alpha(t) \leqslant \rho_{n_{0}}$ for $t \in[0, T]$ with

$$
q(t) f(t, \alpha(t)) \geqslant \alpha^{\prime}(t) \quad \text { for } t \in(0, T)
$$

and

$$
q(t) f(t, y) \geqslant \alpha^{\prime}(t) \text { for }(t, y) \in(0, T) \times\{y \in(0, \infty): y<\alpha(t)\} .
$$

If in addition to (2.23) assume the following holds:

$$
f(\cdot, y) \text { is nondecreasing on }(0, T / 3) \text { for each fixed } y \in(0, \infty) \text {. }
$$

Then (2.5) is satisfied. This follows from (2.24) if $t \in[T / n, T)$, whereas if $t \in(0, T / n)$ then (2.24) and (2.26) yield

$$
q(t) f\left(\frac{T}{n}, \alpha(t)\right) \geqslant q(t) f(t, \alpha(t)) \geqslant \alpha^{\prime}(t) .
$$


In addition it is easy to check that (2.18) also holds.

Combining the above with Theorem 2.1 and Remark 2.2 gives the following existence result.

THEOREM 2.3 Let $n_{0} \in\{3,4, \ldots\}$ be fixed and suppose (2.2), (2.3), (2.7), (2.8), (2.19), (2.23) and (2.26) hold. Then (2.1) has a solution $y \in C[0, T] \cap C^{1}(0, T]$ with $y(t)>0$ for $t \in(0, T]$.

Remark 2.3 In Theorem 2.3 we could replace (2.7), (2.8) with (2.17).

Remark 2.4 One could replace (2.26) in Theorem 2.3 with the more general condition: there exists $\delta \in(0, T / 3)$ with $f(\cdot, y)$ nondecreasing on $(0, \delta)$ for each fixed $y \in(0, \infty)$.

Looking at Theorem 2.1 and Theorem 2.3 we see that the main difficulty when discussing examples is constructing the $\beta_{n}$ in (2.6) (and (2.19)). As a result we present a theorem which removes (2.6) (and (2.19)) and replaces it with an easy verifiable condition. We first present the result in its full generality.

THEOREM 2.4 Let $n_{0} \in\{3,4, \ldots\}$ be fixed and suppose (2.2)-(2.5) hold. Also assume

$$
\left\{\begin{array}{l}
|f(t, y)| \leqslant g(y)+h(y) \text { on }[0, T] \times(0, \infty) \text { with } \\
g>0 \text { continuous and nonincreasing on }(0, \infty) \\
\text { and } h \geqslant 0 \text { continuous on }[0, \infty) .
\end{array}\right.
$$

Also suppose there exists a constant $M>0$ with $G^{-1}(M)>$ $\sup _{t \in[0, T]} \alpha(t)$ and with

$$
\int_{0}^{T} q(x) \mathrm{d} x<\int_{0}^{M} \frac{\mathrm{d} s}{\left[1+\left(h\left(G^{-1}(s)\right) / g\left(G^{-1}(s)\right)\right)\right]}
$$

holding; here $G(z)=\int_{0}^{z} \mathrm{~d} u / g(u)$ (note $G$ is an increasing map from $[0, \infty)$ onto $[0, \infty)$ with $G(0)=0)$. Then $(2.1)$ has a solution $y \in C[0, T] \cap C^{\prime}(0, T]$ with $y(t) \geqslant \alpha(t)$ for $t \in[0, T]$. 
Proof Choose $c>0, c<M$ with

$$
\int_{0}^{T} q(x) \mathrm{d} x<\int_{\varsigma}^{M} \frac{\mathrm{d} s}{\left[1+\left(h\left(G^{-1}(s)\right) / g\left(G^{-1}(s)\right)\right)\right]} .
$$

Let $m_{0} \in\{3,4, \ldots\}$ be chosen so that $G\left(\rho_{m_{0}}\right)<c$ and without loss of generality assume $m_{0} \leqslant n_{0}$. Let $\alpha_{n}$ be as in Theorem 2.1 and again we examine $(2.9)^{n}$ with

$$
f_{n}^{*}(t, y)=\left\{\begin{array}{l}
f\left(\frac{T}{n}, G^{-1}(M)\right), \quad y \geqslant G^{-1}(M) \text { and } 0 \leqslant t \leqslant \frac{T}{n} \\
f\left(t, G^{-1}(M)\right), \quad y \geqslant G^{-1}(M) \text { and } \frac{T}{n} \leqslant t \leqslant T \\
f\left(\frac{T}{n}, y\right), \quad \alpha_{n}(t) \leqslant y \leqslant G^{-1}(M) \text { and } 0 \leqslant t \leqslant \frac{T}{n} \\
f(t, y), \quad \alpha_{n}(t) \leqslant y \leqslant G^{-1}(M) \text { and } \frac{T}{n} \leqslant t \leqslant T \\
f\left(t, \alpha_{n}(t)\right), \quad y \leqslant \alpha_{n}(t) \text { and } \frac{T}{n} \leqslant t \leqslant T \\
f\left(\frac{T}{n}, \alpha_{n}(t)\right), \quad y \leqslant \alpha_{n}(t) \text { and } 0 \leqslant t \leqslant \frac{T}{n} .
\end{array}\right.
$$

As in Theorem $2.1,(2.9)^{n}$ has a solution $y_{n}$ with

$$
y_{n}(t) \geqslant \alpha_{n}(t) \geqslant \alpha(t) \text { for } t \in[0, T] .
$$

Next we show

$$
y_{n}(t)<G^{-1}(M) \text { for } t \in[0, T] \text {. }
$$

Suppose (2.30) is false, then since $y_{n}(0)=\rho_{n}$ there exists $\tau_{1}<\tau_{2} \in[0, T]$ with

$$
\begin{aligned}
& \rho_{n} \leqslant y_{n}(t) \leqslant G^{-1}(M) \\
& \quad \text { for } t \in\left(\tau_{1}, \tau_{2}\right), y_{n}\left(\tau_{1}\right)=\rho_{n} \text { and } y_{n}\left(\tau_{2}\right)=G^{-1}(M) .
\end{aligned}
$$


Now for $t \in\left(\tau_{1}, \tau_{2}\right)$ we have

$$
f_{n}^{*}\left(t, y_{n}(t)\right) \leqslant g\left(y_{n}(t)\right)\left\{1+\frac{h\left(y_{n}(t)\right)}{g\left(y_{n}(t)\right)}\right\}
$$

since if $t \in(0, T / n)$ then $f_{n}^{*}\left(t, y_{n}(t)\right)=f\left(T / n, y_{n}(t)\right) \leqslant g\left(y_{n}(t)\right)+$ $h\left(y_{n}(t)\right)$, whereas if $t \in[T / n, T)$ then $f_{n}^{*}\left(t, y_{n}(t)\right)=f\left(t, y_{n}(t)\right) \leqslant$ $g\left(y_{n}(t)\right)+h\left(y_{n}(t)\right)$. Thus

$$
\frac{y_{n}^{\prime}(t)}{g\left(y_{n}(t)\right)} \leqslant q(t)\left\{1+\frac{h\left(y_{n}(t)\right)}{g\left(y_{n}(t)\right)}\right\} \quad \text { for } t \in\left(\tau_{1}, \tau_{2}\right) .
$$

Let

$$
v_{n}(t)=\int_{0}^{y_{n}(t)} \frac{\mathrm{d} u}{g(u)}=G\left(y_{n}(t)\right)
$$

and so

$$
v_{n}^{\prime}(t) \leqslant q(t)\left\{1+\frac{h\left(G^{-1}\left(v_{n}(t)\right)\right)}{g\left(G^{-1}\left(v_{n}(t)\right)\right)}\right\} \text { for } t \in\left(\tau_{1}, \tau_{2}\right) .
$$

Integrate from $\tau_{1}$ to $\tau_{2}$ to obtain

$$
\begin{aligned}
& \int_{\epsilon}^{v_{n}\left(\tau_{2}\right)} \frac{\mathrm{d} s}{\left[1+\left(h\left(G^{-1}(s)\right) / g\left(G^{-1}(s)\right)\right)\right]} \\
& \quad \leqslant \int_{G\left(\rho_{n}\right)}^{v_{n}\left(\tau_{2}\right)} \frac{\mathrm{d} s}{\left[1+\left(h\left(G^{-1}(s)\right) / g\left(G^{-1}(s)\right)\right)\right]} \\
& \quad \leqslant \int_{0}^{T} q(s) \mathrm{d} s<\int_{\epsilon}^{M} \frac{\mathrm{d} s}{\left[1+\left(h\left(G^{-1}(s)\right) / g\left(G^{-1}(s)\right)\right)\right] .}
\end{aligned}
$$

Consequently $v_{n}\left(\tau_{2}\right)<M$ so $y_{n}\left(\tau_{2}\right)<G^{-1}(M)$. This is a contradiction. Thus (2.30) holds and so

$$
\alpha(t) \leqslant \alpha_{n}(t) \leqslant y_{n}(t)<G^{-1}(M) \text { for } t \in[0, T] .
$$

Essentially the same reasoning as in Theorem 2.1 from (2.16) onwards completes the proof.

We also have the following result. 
THEOREM 2.5. Let $n_{0} \in\{3,4, \ldots\}$ be fixed and suppose (2.2), (2.3), (2.23), (2.26) and (2.27) hold. In addition assume there is a constant $M>0$ with

$$
\int_{0}^{T} q(x) \mathrm{d} x<\int_{0}^{M} \frac{\mathrm{d} s}{\left[1+\left(h\left(G^{-1}(s)\right) / g\left(G^{-1}(s)\right)\right)\right]}
$$

holding; here $G(z)=\int_{0}^{z} \mathrm{~d} u / g(u)$. Then (2.1) has a solution $y \in C[0, T] \cap C^{1}(0, T]$ with $y(t)>0$ for $t \in[0, T]$.

Proof This follows immediately from Theorem 2.4 once we show

$$
G^{-1}(M)>\alpha(t) \quad \text { for each } t \in[0, T]
$$

( $\alpha$ is described after (2.23)). Suppose this is false. Then since $\alpha(0)=0$ there exists $\tau_{1}<\tau_{2} \in[0, T]$ with

$$
\begin{array}{ll}
0 \leqslant \alpha(t) \leqslant G^{-1}(M) & \text { for } t \in\left(\tau_{1}, \tau_{2}\right), \alpha\left(\tau_{1}\right)=0 \\
& \text { and } \alpha\left(\tau_{2}\right)=G^{-1}(M) .
\end{array}
$$

Notice (2.23) (see (2.24)) implies

$$
\alpha^{\prime}(t) \leqslant q(t) f(t, \alpha(t)) \quad \text { for } t \in\left(\tau_{1}, \tau_{2}\right),
$$

so we have

$$
\frac{\alpha^{\prime}(t)}{g(\alpha(t))} \leqslant q(t)\left\{1+\frac{h(\alpha(t))}{g(\alpha(t))}\right\} \text { for } t \in\left(\tau_{1}, \tau_{2}\right)
$$

Let

$$
v(t)=\int_{0}^{\alpha(t)} \frac{\mathrm{d} u}{g(u)}=G(\alpha(t)),
$$

so

$$
v^{\prime}(t) \leqslant q(t)\left\{1+\frac{h\left(G^{-1}(v(t))\right)}{g\left(G^{-1}(v(t))\right)}\right\} \quad \text { for } t \in\left(\tau_{1}, \tau_{2}\right)
$$


Integrate from $\tau_{1}$ to $\tau_{2}$ to obtain

$$
\begin{aligned}
& \int_{0}^{v\left(\tau_{2}\right)} \frac{\mathrm{d} s}{\left[1+\left(h\left(G^{-1}(s)\right) / g\left(G^{-1}(s)\right)\right)\right]} \\
& \quad \leqslant \int_{0}^{v\left(\tau_{2}\right)} \frac{\mathrm{d} s}{\left[1+\left(h\left(G^{-1}(s)\right) / g\left(G^{-1}(s)\right)\right)\right]} \\
& \quad \leqslant \int_{0}^{T} q(s) \mathrm{d} s<\int_{0}^{M} \frac{\mathrm{d} s}{\left[1+\left(h\left(G^{-1}(s)\right) / g\left(G^{-1}(s)\right)\right)\right]}
\end{aligned}
$$

Thus $v\left(\tau_{2}\right)<M$, so $\alpha\left(\tau_{2}\right)<G^{-1}(M)$, a contradiction.

Remark 2.5 In Theorem 2.5 we could replace (2.23), (2.26) with Eqs. (2.4) and (2.18).

Next we present some examples which illustrate how easily the theory is applied in practice.

Example 2.1 The initial value problem

$$
\left\{\begin{array}{l}
y^{\prime}=y^{-\alpha}+y^{\beta}+A, \quad 0<t<T(<\infty) \\
y(0)=0, \quad \alpha, \beta>0, \quad A \geqslant 0
\end{array}\right.
$$

has a solution $y \in C[0, T] \cap C^{\mathrm{l}}(0, T]$ with $y(t)>0$ for $t \in(0, T]$ if

$$
T<\int_{0}^{\infty} \frac{\mathrm{d} s}{1+[(\alpha+1) s]^{(\beta+\alpha) /(\alpha+1)}+A[(\alpha+1) s]^{\alpha /(\alpha+1)}} .
$$

To see this we will apply Theorem 2.5 with

$$
n_{0}=3, \quad q=1, \quad g(y)=y^{-\alpha}, \quad h(y)=y^{\beta}+A,
$$

together with

$$
\rho_{n}=\frac{1}{n} \text { and } k_{0}=3^{\alpha} \text {. }
$$

Clearly (2.2), (2.3), (2.26) and (2.27) hold. Also for $n \in\{3,4, \ldots\}$, $(T / n) \leqslant t \leqslant T$ and $0<y \leqslant \rho_{n}$ we have

$$
q(t) f(t, y) \geqslant y^{-\alpha} \geqslant n^{\alpha} \geqslant 3^{\alpha},
$$


so (2.23) is satisfied. From (2.34) there exists $M>0$ with

$$
T<\int_{0}^{M} \frac{\mathrm{d} s}{1+[(\alpha+1) s]^{(\beta+\alpha) /(\alpha+1)}+A[(\alpha+1) s]^{\alpha /(\alpha+1)}},
$$

so now (2.32) holds with this $M$ since

$$
G(z)=\frac{z^{\alpha+1}}{\alpha+1}, \text { so } G^{-1}(z)=[(\alpha+1) z]^{1 / \alpha+1} \text {. }
$$

Existence of a solution to (2.33) is now guaranteed from Theorem 2.5. Example 2.2 The initial value problem

$$
\left\{\begin{array}{l}
y^{\prime}=\left(\frac{t^{\alpha}}{y^{0}}+A y^{\beta}-\lambda\right), 0<t<T(<\infty) \\
y(0)=0,
\end{array}\right.
$$

with $\alpha>0, \theta \geqslant 0, \lambda>0, \alpha \geqslant \theta, 0 \leqslant \beta<1, A \geqslant 0$ has a solution $y \in C[0, T] \cap C^{\prime}(0, T]$ with $y(t)>0$ for $t \in(0, T]$.

To see this we will apply Theorem 2.3 with

$$
n_{0}=3, \quad q=1, \quad g(y)=\frac{T^{\alpha}}{y^{0}}, \quad h(y)=A y^{\beta}+\lambda,
$$

together with

$$
\rho_{n}=\left(\frac{T^{\alpha}}{(\lambda+1) n^{\alpha}}\right)^{1 / 0} \text { and } k_{0}=1
$$

Clearly (2.2), (2.3), (2.8) and (2.26) are satisfied. Also for $n \in\{3,4, \ldots\},(T / n) \leqslant t \leqslant T$ and $0<y \leqslant \rho_{n}$ we have

$$
q(t) f(t, y) \geqslant \frac{t^{\alpha}}{y^{0}}-\lambda \geqslant\left(\frac{T}{n}\right)^{\alpha} \frac{1}{\rho_{n}^{0}}-\lambda=(\lambda+1)-\lambda=1 .
$$


Thus (2.23) holds. It remains to check (2.7) and (2.19). Let

$$
\beta_{n}(t)=a t+\rho_{n}
$$

where $a>0$ is chosen so that

$$
\frac{T^{\alpha-\theta}}{a^{\theta}}+A\left(a T+\rho_{3}\right)^{\beta}-\lambda-a \leqslant 0 \text { and } 1+A\left(a T+\rho_{3}\right)^{\beta}-a \leqslant 0 ;
$$

the existence of an $a>0$ so that (2.36) holds is immediate since $0 \leqslant \beta<1$. Clearly $(2.7)$ is true. Also if $n \in\{3,4, \ldots\}$ and $(T / n) \leqslant t \leqslant T$ we have

$$
\begin{aligned}
q(t) f\left(t, \beta_{n}(t)\right)-\beta_{n}^{\prime}(t) & \leqslant\left[\frac{t^{\alpha}}{[a t]^{\theta}}+A\left(a t+\rho_{n}\right)^{\beta}-\lambda\right]-a \\
& \leqslant \frac{T^{\alpha-\theta}}{a^{\theta}}+A\left(a T+\rho_{3}\right)^{\beta}-\lambda-a \\
& \leqslant 0
\end{aligned}
$$

whereas if $n \in\{3,4, \ldots\}$ and $0<t<(T / n)$ we have

$$
\begin{aligned}
q(t) f\left(\frac{T}{n}, \beta_{n}(t)\right)-\beta_{n}^{\prime}(t) & =\left[\left(\frac{T}{n}\right)^{\alpha} \frac{1}{\rho_{n}^{\theta}}+A\left(a t+\rho_{n}\right)^{\beta}-\lambda\right]-a \\
& \leqslant\left[(\lambda+1)+A\left(a T+\rho_{3}\right)^{\beta}-\lambda\right]-a \\
& =1+A\left(a T+\rho_{3}\right)^{\beta}-a \\
& \leqslant 0 .
\end{aligned}
$$

Thus (2.19) holds. Existence of a solution to (2.35) is now guaranteed from Theorem 2.3.

\section{References}

[1] Agarwal, R.P. and O'Regan, D. (2001) Existence theory for single and multiple solutions to singular positone boundary value problems. Jour. Differential Equations, 175, 393-414. 
[2] Agarwal, R.P., O'Regan, D. and Wong, P.J.Y. (1999). Positive Solutions of Differential, Difference and Integral Equations. Kluwer Acad. Publ., Dordrecht.

[3] Bobisud, L.E. and O'Regan, D. (1988). Existence of solutions to some singular initial value problems. Jour. Math. Anal. Appl., 133, 214-230.

[4] Lakshmikantham, V. and Leela, S. (1969). Differential and Integral Inequalities, Vol. 1. Academic Press, New York.

[5] Nkashama, M.N. (1989). A generalized upper and lower solution method and multiplicity results for nonlinear first order ordinary differential equations. Jour. Math. Anal. Appl.. 140, 381-395.

[6] Piccinini, L.C., Stampacchia, G. and Vidossich, G. (1984). Ordinary Differential Equations in $\mathbf{R}^{n}$. Springer, New York. 


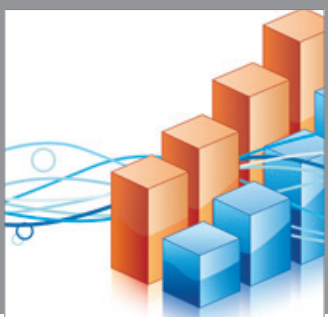

Advances in

Operations Research

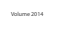

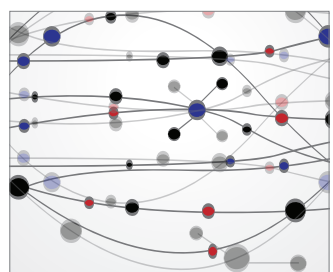

\section{The Scientific} World Journal
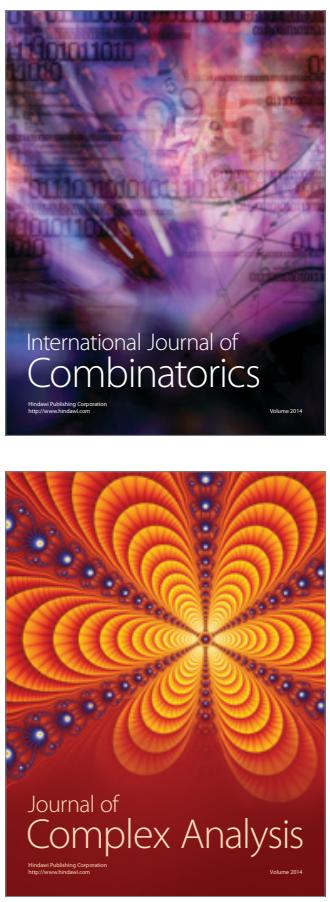

International Journal of

Mathematics and

Mathematical

Sciences
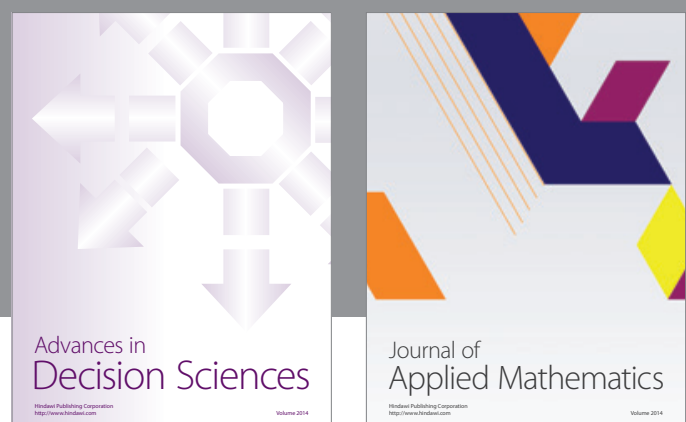

Journal of

Applied Mathematics
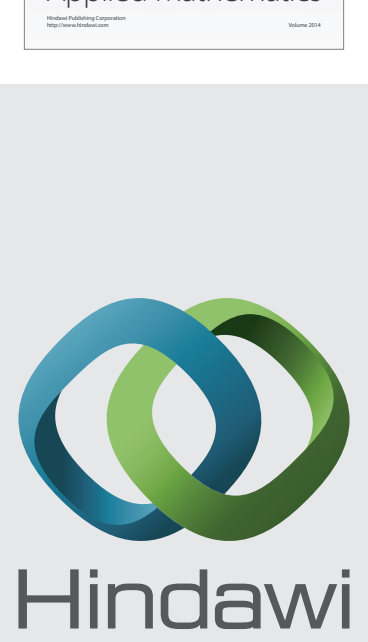

Submit your manuscripts at http://www.hindawi.com
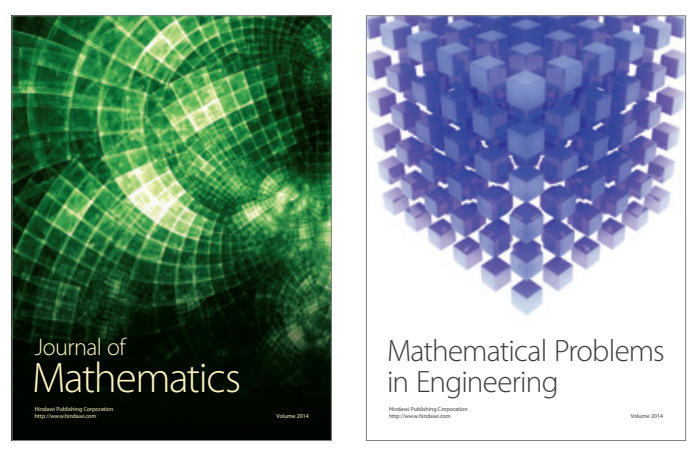

Mathematical Problems in Engineering
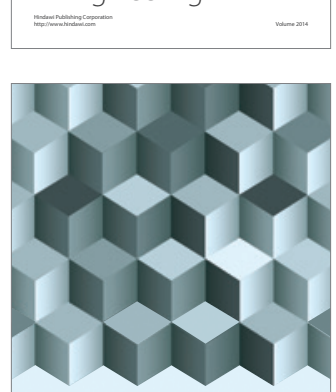

Journal of

Function Spaces
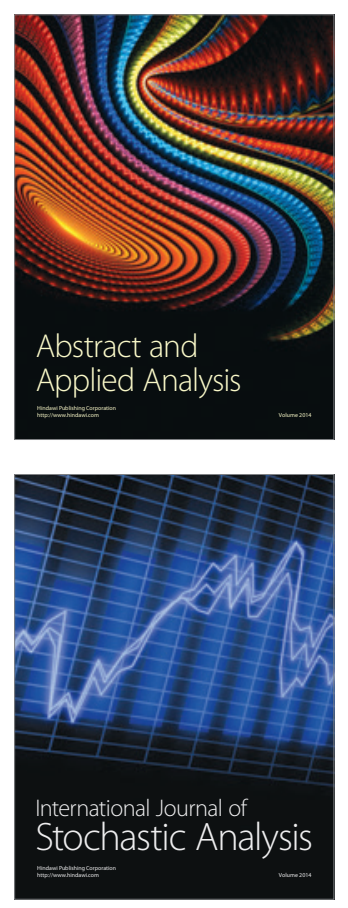

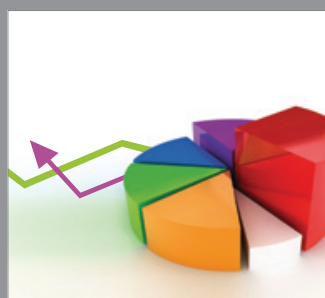

ournal of

Probability and Statistics

Promensencen
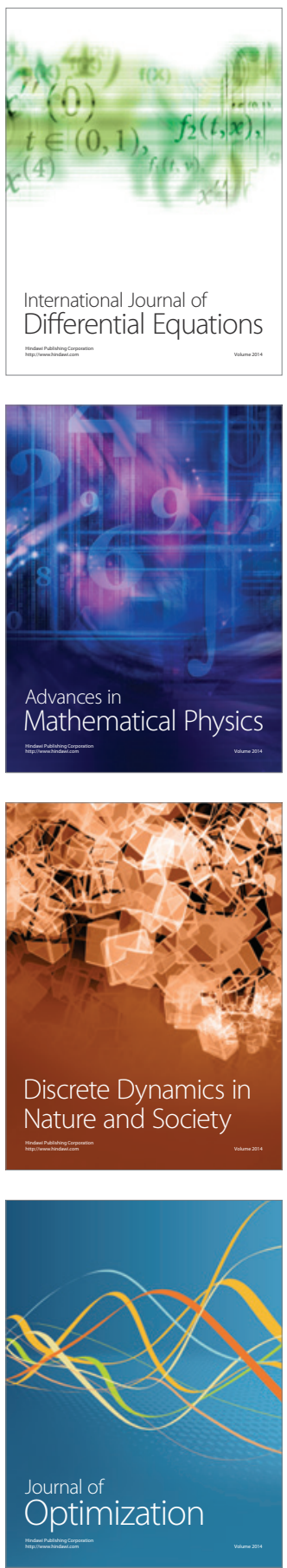